\title{
A Regional Approach to Promoting Improved Care of Multiples
}

\author{
Patricia Maxwell Malmstrom \\ Twin Services Consulting, USA
}

\begin{abstract}
ive births of multiples in the U.S. rose $35 \%$ from 87,700 in -1988 to 118,295 in 1998 . This increase presents public health issues due to the elevated health and psychosocial risks that accompany multiple birth. However, health and social service providers and educators are poorly prepared to address the specific needs of the multiple birth population. The Twin Service Network Project therefore developed regional networks of multiple birth training and resources in California to address this problem. Results indicate that these can substantially improve the care available to multiples. The project's integrated package of training and parenting education materials is available to other regions to assist in such efforts.
\end{abstract}

Live births of multiples in the U.S. rose $35 \%$ from 87,700 in 1988 to 118,295 in 1998 (National Center for Health Statistics, 1998, 2000). This increase presents public health issues due to the elevated health and psychosocial risks that accompany a multiple birth (Keith et al., 1995; Malmstrom \& Poland, 1999). Multiples account for a disproportionately large fraction of the populations in neonatal intensive care units (NICU), foster care, and special education classes (California Department of Social Services, $1994^{1}$; Krall \& Feinstein, 1991).

Annual aggregate health care costs associated with U.S. multiple births exceed $\$ 1.5$ billion, 35\% of which is billed to Medicare/Medicaid for the care of low income families (Lewit et al., 1995²). Therefore, families with multiples are an easily identifiable population to target to reduce perinatal health risks and expenditures and improve family functioning. Since its establishment in 1978, the independent, non-profit making Twin Services, Inc., based in Alameda County, California, has seen that the health and social service providers and educators are poorly prepared to address this issue. Countrywide requests for information and resources from parents and professionals made it clear that few care givers had any training regarding the needs and care of multiples.

Misconceptions led to serious consequences. The obstetrician for a mother of still born multiples failed to refer her to a high risk clinic because he did not believe that multiple pregnancy involved high risk. A pathologist reported fetal deaths among monozygotic multiples which might have been averted had their chorionicity been determined and the pregnancy more closely monitored. Some mothers reported that they had been discouraged from breastfeeding their multiples by nurses who told them they would not be able to produce enough milk. The Women Infants and Children (WIC) program, which provides food for low income pregnant and lactating women, does not increase allotments for women expecting or breastfeeding multiples.

The Twin Service Network Project (1992-97) aimed to transfer understanding of multiple birth needs and preventive care to service providers throughout California by facilitating training for professionals and mobilizing community resources to address the special needs of multiples.

\section{$\overline{\text { Method }}$}

Regional networks were launched in northern and southern California. Regional advisory boards were composed of representatives of community- and hospital-based programs, which ensured comprehensive representation of relevant health and social services. Statistics on multiple births and deaths and related public health issues were gathered. California maternal and child health data were analyzed, as were regional data from county sources together with a survey of 39 administrators of targeted East Bay programs. Findings were disseminated statewide among health and family service programs.

The pilot phase of the project (1992-95) targeted programs in Alameda and Contra Costa Counties, California, the birthplace annually of about 600 multiples. Eight representatives of East Bay programs, which had used Twin Services' programs, volunteered for the advisory board. They, in turn, recruited twelve more members. Members of the advisory board met bi-monthly to plan and assist with data collection and evaluation. They also helped prepare and implement the project's training program.

A training day, Twinshock Training: Promoting Preventive Care of Multiples, was prepared, tested, and revised in the East Bay. The program and its accompanying workbook, Relieving Twinshock: A Guide for Helping Families with Multiples, provide an overview of multiple birth statistics, biology, health and psychosocial risks. They encourage the integration of multiple-birth-specific protocols of care and preventive interventions into existing health, social service and education programs. To encourage follow-up activities by trainees, packets of reproducible

Correspondence to: Patricia Malmstrom, Director, Twin Services Consulting, P.O. Box 10066, Berkeley CA 94709, USA.

E-mail: twinservices@juno.com

Manuscript received 12 July 2000, accepted I January 200 I. 
materials, including a check-list of multiple birth needs and an assessment protocol, were provided.

Existing telephone helplines were used to give parents access to multiple birth information and resources. The project's service was based upon Twin Services' national helpline, the TWINLINE®, which had already been in use since 1978. Its professional counseling staff offered health and parenting education and referral to other services for families expecting and rearing multiples. Information and advice were also offered to professionals. All callers were provided with supplementary information from Twin Services' Twin Care Handout Collection, a series of articles regarding key multiple birth issues from pregnancy to adolescence (Malmstrom \& Biale, 1990).

The project's training curriculum was later implemented in California's two largest metropolitan areas, the San Francisco Bay Area and Los Angeles County. These two regions together are the birthplace of $14 \%$ of California's multiples.

\section{Results and Discussion}

California Maternal and Child Health data showed the elevated risks of multiple births in California between 1986-89. Low birth weight (< 2500 grams) and very low birth weight $(<1500$ grams) occurred about ten times more frequently for multiples than for the single born. Infant death was four to five times as likely for multiples. Cesarean section delivery was three times more common among multiple births. Project reports publicized the needs of multiples and project training activities to over 1900 perinatal health and service providers statewide.

Professionals proved to be quite willing to serve on project advisory boards. One pediatric nurse explained, "Multiples make up ten percent of our patients and we've never had a full day training about them". A perinatologist commented, "I'm sick of saving these fragile babies and having them come back [to the hospital] battered". Advisory board meetings became regional forums linking professionals across programs, disciplines and county boundaries. Participants used their combined expertise to illuminate issues, disseminate project reports, adapt curricula, and to convince program administrators to include such training in their programs for professionals.

The provision of voluntary services, goods and meeting space contributed one fourth of project costs.

Multiple birth statistics were shown to be underused. In Alameda County the practice of excluding multiple births before analyzing trends resulted in the elimination of multiples from the planning process and contributed to provider ignorance. Later, multiple births were included and shown to be one of the county's five highest obstetric risks.

A "snap shot" of multiple birth care prior to project training was developed from a survey of thirty-nine administrators of East Bay programs. Twenty of the administrators represented health and social service agencies, fourteen were from hospital-based programs, four from health clinics/medical groups, and one was from a child care center. None could provide the actual numbers of multiples served by their programs because of lack of data, or of access to it. Administrators' estimates for the prior year ranged from a high of 51-100 multiples in two hospitals to a low of $1-10$ in nineteen health and social service programs.

East Bay families presented most of the elevated health risks associated with multiples, including maternal complications, fetal and neonatal complications, disability and death. Few of the programs addressed multiple birth needs specifically. Nine administrators had used a staff member who had had some personal experience of multiples as a "multiple birth specialist".

Twenty of the 30 administrators who did not have a "multiple birth specialist" reported that they routinely referred multiple birth families to Twin Services. Others indicated that the small size of the population did not justify special staff, their whole staff attended to all clients, or that they hadn't focused on the issue. Ten reported that no funds were available for such a position. Participation in the survey heightened administrators' awareness of the needs of multiples in their care and the project's training.

Twinshock Training reached over 300 staff from nineteen East Bay programs in eleven sessions held during 1993-95. In turn, those trainees trained an estimated 600 of their colleagues. The day-long curriculum included discussion of adjustments that trainees could make to their specific program protocols. Sometimes the curriculum had to be shortened to a half day. Although the packets of training materials facilitated follow up, the full-day sessions were clearly regarded as preferable.

Six months later, fifty-three of the original trainees completed follow-up questionnaires. Respondents indicated an almost two-fold increase in their provision of multiple birth-specific care. Use of Twin Services' parenting education materials and referral services had more than doubled. Special assessment and referral to relevant services had almost doubled. Initiatives in educating colleagues showed a more than three-fold increase (see Figure 1). All respondents reported a more positive response to multiples. A public health nurse commented, "The training had a tremendous impact on our staff's awareness of multiple birth. I really enjoyed the training. It's so practical". Another wrote, "My [new] knowledge came in handy one week after the training when I saw a mother who had just lost one of her twins".

A two-fold increase in provider usage of the project's telephone helpline, and a follow-up sampling of outcomes for parents, indicate the effectiveness of this strategy. More than 600 East Bay providers called during the pilot phase of the project, 1992-95. As word of the service spread, the numbers of calls more than doubled. Helpline services reached 1,377 East Bay parents. The combination of health related and practical needs for which parents called are illustrated in Table 1. Twenty clients, including twelve $(60 \%)$ with low income, were interviewed individually following use of this service. All had received referrals to community resources. Seven had received help about post natal health issues and five were advised about family functioning. All recorded improvements. Both low income and other parents said they experienced reduced stress after they shared their concerns with a counselor. The most common 
Figure 1: Professionals Reporting Improved Multiple Birth Services ( $\mathbf{N}=\mathbf{5 3}$ )

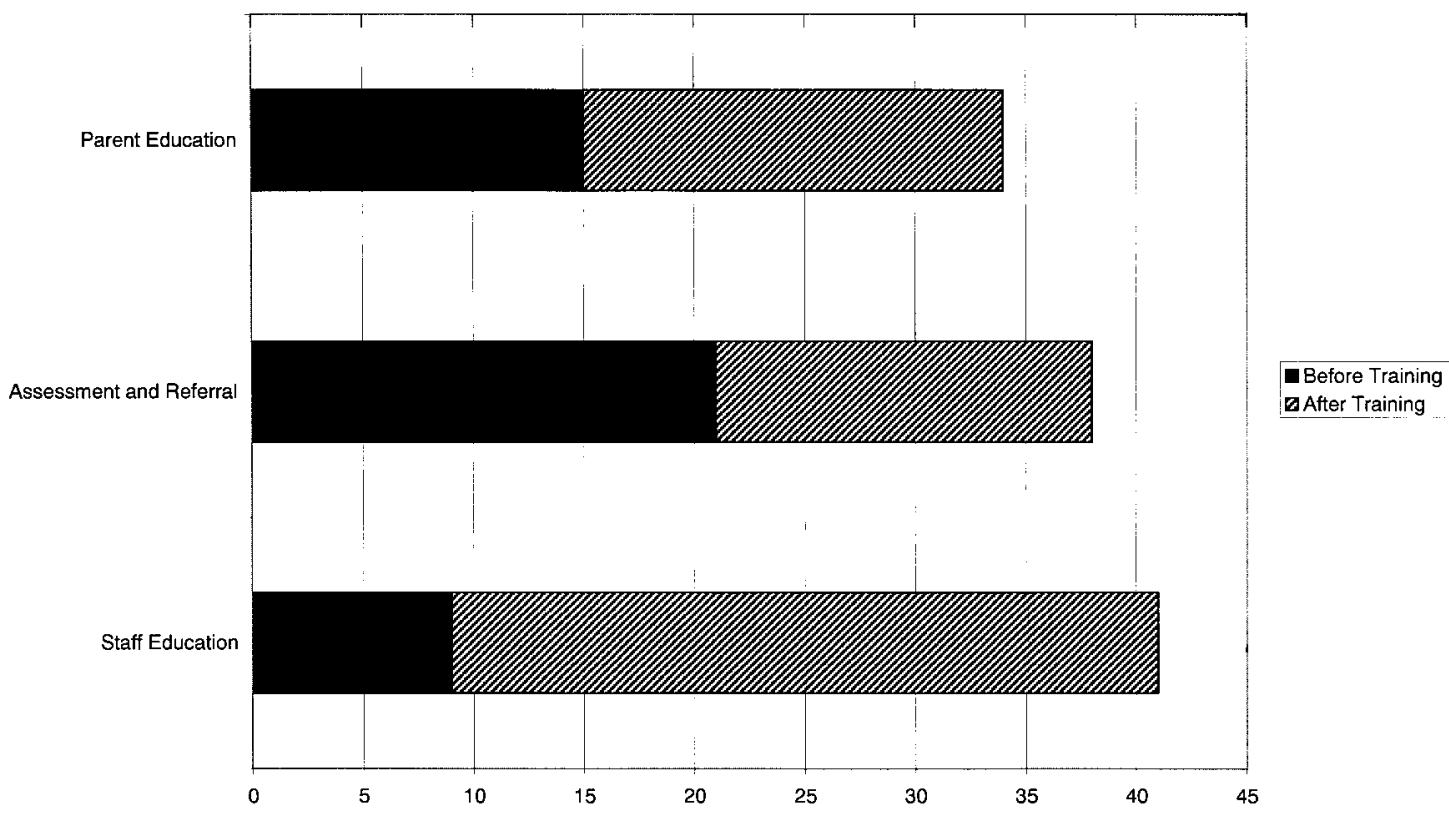

Figure 1

Professionals reporting increased multiple birth services after training $(N=53)$.

improvement in family functioning was the development of a more efficient household schedule.

Results indicate that parents with multiples need help to follow up referrals. As the project counseling staff had to reduce their hours (because of budget restraints) they were unable to assist callers with this. The twelve low income interviewees had received a total of 51 referrals and the other eight, a total of 22 referrals. In both groups over half had not been followed up. There is no clear evidence to explain this low follow-up rate. Parents may have received service elsewhere or received satisfactory service from the first resource contacted. It is most likely that many parents were too overwhelmed to follow up. Anecdotal data indicate that parents suffered extraordinary sleep deprivation, which

\section{Table 1}

Multiple Birth Parents' Health Issues and Practical Needs

Sample calls to the project's twinline during March \& April 1995

1 developmental delays; double stroller

2 clinical depression; respite

3 infant digestive disorder, high chairs \& diapers

4 home-care of one low birth weight infant; hospital care of co-twin; transportation to hospital

5 gestational diabetes \& preterm labor; congenital anomaly in one infant

6 low birth weight infants; mother pumping breastmilk for bottle feeding

7 maternal blackouts, double stroller, car seats for multiples and two year old; toddler clothing

8 maternal death; grief counseling; financial assistance; diapers $\&$ formula diminished both memories and initiative. Often callers did not remember their initial call, nor the reason for it. One new mother could not recall the birth date of her twins. This reinforces the prior experience of Twin Services that stressed parents benefit from active help to link them to community resources (Malmstrom et al., 1986).

Over 160 providers participated in the launch of regional networks in the San Francisco Bay Area and in Los Angeles County during the final two project years, 1995-97. In response to requests from parent clubs, a chapter was added to the training manual to help volunteers who had no relevant professional experience to conduct a training session. Copies were distributed to targeted health and perinatal service programs throughout California to encourage replication of the Twin Service Network.

Project participants have become effective advocates for multiple birth families within California's programs for infants, children and families. Their accomplishments include the addition of a chapter on multiple birth care in the perinatal service manual published by the California Department of Health's Maternal and Child Health Branch. Two East Bay programs have submitted proposals to pilot home visiting services and in-home help for low income families with multiples. Eight articles from Twin Services' parenting education series were translated into Spanish. For the first time, multiple birth was made the focus of the East Bay's annual perinatal conference attended by 200 professionals.

\section{Conclusion}

Professional care providers have a tremendous opportunity to improve the health and welfare of multiples by giving 
them the systematic and sustained attention they deserve. The results of this project indicate that regional networks of representatives of hospital- and community-based programs are effective mechanisms for training health and social service providers. When alerted to the special needs of multiple birth infants and their families, many professionals will develop appropriate services. The project's model for developing regional networks of multiple birth training services and community resources, and its integrated package of training and parenting education materials can assist in these efforts.

\section{Acknowledgments}

The Twin Service Network Project was supported in part by project $\mathrm{MCH} \# 067920-01-0$ from the Maternal and Child Health program (Title V, Social Security Act) Health Resources and Services Administration, U.S. Department of Health and Human Services, and by grants from the California Wellness Foundation, the San Francisco Foundation, and the Bay Area Chapter of the March of Dimes.

\section{Footnotes}

1 Since multiple births are not identified per se in this database the count of children with the same last name and date of birth ages $0-3$ was used as an approximate surrogate. Their representation in foster care placement was $8 \%$ higher than that of all children ages $0-3$.

2 The proportion of low birth weight costs represented by multiple births were calculated and then adjusted for inflation to determine costs in 1995 dollars.

\section{$\overline{\text { References }}$}

California Department of Social Services. (1994). Foster Care Children Who Entered Placement During Calendar Year 91, 92, 93 and Counts of Same Last Names, Serial Number and Birthdate. Foster Care Information System \# 94-34.

Keith, L.G., Papiernik, E., Keith, D.M., \& Luke, B. (Eds). (1995). Multiple pregnancy: Epidemiology, Gestation \& Perinatal Outcome. New York: Parthenon.

Krall, V., \& Feinstein, S.C. (1991). Psychological Development of High Risk Multiple Birth Children. Chur, Switzerland: Harwood Academic.

Lewit , E.M., Baker, L.S., Corman, H., \& Shiono, P. (1995). The direct cost of low birth weight. The Future of Children 5, 35-56. Los Altos, CA: Center for the Future of Children.

Malmstrom, P.M., \& Biale, R. (1990). An Agenda for Meeting the Special Needs of Multiple Birth Families. Acta Geneticae Medicae et Gemellologiae 39, 507-514.

Malmstrom, P.M., \& Poland, J. (1999). The Art of Parenting Twins. New York: Ballatine.

Malmstrom, P.M., Wedge, M.W., Faherty, T., \& Wagner, P. (1986). Respite care: A lifeline for low-income multiple birth families. [Abstract] Acta Geneticae Medicae et Gemellologiae 35, 207.

National Center for Health Statistics (1998). Monthly vital statistics report (supplement) 46, 11, 21-22. Hyattsville, Maryland.

National Center for Health Statistics (March 28, 2000). Births in the U.S. increase for the first time since 1990. News Releases and Fact Sheets, HHS News. Hyattsville, Maryland. 